BULL. AUSTRAL. MATH. SOC.

$46 \mathrm{E} 40,46 \mathrm{GlO}, 47 \mathrm{~B} 38$

VOL. 36 (1987) 267-278.

\title{
OPERATORS ON LOCÁLLY CONVEX SPACES \\ OF VECTOR-VALUED CONTINUOUS FUNCTIONS
}

A. GARCía LóPez

Let $E$ and $F$ be locally convex spaces and let $K$ be a compact Hausdorff space. $C(K, E)$ is the space of all $E$-valued continuous functions defined on $K$, endowed with the uniform topology.

Starting from the well-known fact that every linear continuous operator $T$ from $C(K, E)$ to $F$ can be represented by an integral with respect to an operator-valued measure, we study, in this paper, some relationships between these operators and the properties of their representing measures. We give special treatment to the unconditionally converging operators.

As a consequence we characterise the spaces $E$ for which an operator $T$ defined on $C(K, E)$ is unconditionally converging if and only if $\left(T f_{n}\right)$ tends to zero for every bounded and converging pointwise to zero sequence $\left(f_{n}\right)$ in $C(K, E)$.

Received 13 october 1986 . This research was partially supported by CAICYT grang $n$. 0338/84.

Copyright Clearance Centre, Inc. Serial-fee code: 0004-9727/87 $\$ A 2.00+0.00$. 


\section{Introduction}

Throughout this paper $K$ is a compact Hausdorff topological space, $\Sigma$ the Borel $\sigma$-field of $K, E$ and $F$ are quasicomplete Hausdorff locally convex spaces, $P_{E}$ and $P_{E}$ saturated families of seminorms defining the topologies of $E$ and $F$ respectively, $C(K, E)$ is the space of all continuous $E$-valued functions defined on $K$, with the uniform convergence topology.

We are interested in operators (= continuous linear operators) $T$ from $C(K, E)$ to $F$ and their operator-valued representing measures. The study of the relationship between an operator and $i$ ts representing measure has been considered by many authors, see for instance [1], [2], [3], [5], [9], [11] or [12]. Some interesting characterisations for several properties of $T$ in terms of properties of $m$ are known when $E$ and $F$ are Banach spaces. In this paper we consider this class of problems in the general case where $E$ and $F$ are locally convex spaces.

The notation and terminology used and not defined can be found in [4] or [8].

Before proceeding further, let us give some definitions and results for reference purpose.

DEFINITION 1. [3] If $m: \Sigma \rightarrow L(E, F)$ is a (finitely additive) operator-valued measure, $q \in P_{F}, p \in P_{E}$ and $A \in \Sigma$ then define

$$
\tilde{m}_{(p, q)}(A)=\sup \left\{q\left(\sum_{i \in \pi} m\left(A_{i}\right) x_{i}\right): \pi \in \Pi(A), \quad x_{i} \in V_{p}\right\}
$$

where $\pi(A)$ denotes the set of disjoint finite $\Sigma$-partitions of $A$ and $V_{p}=\{x \in E: p(x) \leq 1\}$.

We say that $m$ has bounded semivariation if for each $q$ in $P_{F}$ there is a $p$ in $P_{E}$ with $\tilde{m}(p, q)(K)$ finite, and we write $p$ q to denote this correspondence. 
THEOREM 2. [3]. If $T: C(K, E) \rightarrow F$ is an operator, then there is a a unique representing measure $m: \Sigma \rightarrow L\left(E, F^{\prime \prime}\right)$ such that

i) $m$ has bounded semivariation

ii) for $x \in E$ and $z^{\prime} \in F^{\prime}, m_{x z^{\prime}},()=.\left\langle m\left(.\left|x, z^{\prime}\right\rangle\right.\right.$ is a finite regular Borel measure

iii) for $f \in C(K, E)$

$$
T(f)=\int_{K} f d m
$$

The reader could consult [3], [11] and [12] for more information about representing measures.

Remarks: It is easy to prove for $f \in C(K, E), A \in \sum$ and $p \sim q$ that

$$
q\left(\int_{A} f d m\right) \leq \tilde{m}(p, q)(A) \sup \{p(f(t)): t \in A\}
$$

If $x \in E$, the vector measure defined from $\Sigma$ with values in $F$ by $m_{x}()=.m()$.$x is the representing measure of the operator$ $T_{x}: C(K) \rightarrow F, T_{x}(\Psi)=T(x \Psi)$; so an easy extension of a classical theorem of Bartle, Dunford and Schwartz (VI.2.1. of [4]), proves that $m(\Sigma) \subseteq L(E, F)$ if and only if $T_{x}$ is a weakly compact operator for every $x \in E$.

\section{The strongly continuous at $\phi$ measures}

In this section we introduce a new concept of semivariation for an operator-valued measure very helpful in characterising some properties of an operator $T$ from $C(K, E)$ to $F$.

DEFINITION 3. For $m: \Sigma \rightarrow L(E, F), q \in P_{F}, B$ a bounded subset of $E$ and $A \in \Sigma$, we define $\tilde{m}_{B q}(A)$ by

$$
\tilde{m}_{B q}(A)=\sup \left\{q\left(\sum_{i \in \pi} m\left(A_{i}\right) x_{i}\right): \pi \in \Pi(A),\left\{x_{i}\right\} \subset B\right\} .
$$

We say that $m$ is strongly continuous at $\phi(s . c . v$.$) if for each bounded$ 
set $B \subseteq E$ and each $q \in P_{F}$

$$
\lim \tilde{m}_{B q}\left(A_{n}\right)=0
$$

for every decreasing sequence $\left(A_{n}\right)+\phi$ in $\Sigma$.

When $E$ and $E$ are Banach spaces, the s.c.v. measures are the $s$-bounded measures of [3], or those with semivariation continuous at $\phi$ (see [2], [5] or [9]), so the representing measure of every compact, weakly compact, absolutely summing, nuclear or unconditionally converging operator possesses this property.

Now we study some properties of $\tilde{m}_{B q}$.

For each $z^{\prime}$ in $F^{\prime}$, let $m_{z^{\prime}}$, be the vector measure, with values in the locally conyex space $\left(E^{\prime}, B\left(E^{\prime}, E\right)\right)$, defined by

$$
\left\langle x, m_{z},(A)\right\rangle=\left\langle m(A) x, z^{\prime}\right\rangle \quad x \in E, A \in \Sigma \text {. }
$$

Whenever $m$ is a representing measure, $m_{z^{\prime}}$ has bounded variation, that is:

$$
\left|m_{z},\right|_{p},(K)=\sup \left\{\sum_{i=1}^{n} p^{\prime}\left(m_{z^{\prime}}\left(A_{i}\right)\right): \quad\left\{A_{i}\right\} \in \Pi(K)\right\}<\infty
$$

for every continuous seminorm $p^{\prime}$ on $E^{\prime}$. Indeed each $\left|m_{z}{ }^{\prime}\right|_{p}$ is a finite positive Borel regular measure on $K$.

It can also easily be shown that the following property holds:

If $B$ is a bounded set in $E, P_{B}$ is the seminorm defined on $E^{\prime}$ by $P_{B}\left(x^{\prime}\right)=\sup \left\{\left|\left\langle x, x^{\prime}\right\rangle\right|: x \in B\right\}$ and $q \in P_{F}$, then

$$
\tilde{m}_{B q}(A)=\sup \left\{\left|m_{z},\right|_{p_{B}}(A): z^{\prime} \in V_{q}^{\circ}\right\}, A \in \Sigma .
$$

PROPOSITION 4. Let $m: \Sigma \rightarrow L(E, F)$ be a representing measure, $B$ a bounded disc (absolutely convex set) in $E$ and $A \in \Sigma$, then:

a) $\tilde{m}_{B q}(A)=\sup \left\{q\left(\int_{A} f d m\right): f \in C(K, E), f(A) \subseteq B\right\}$ for $q \in P_{F}$,

b) $\left|m_{z^{\prime}}\right|_{p_{B}}(A)=\sup \left\{\left|<\int_{A} f d m, z^{\prime}>\right|: f \in C(K, E), f(A) \subseteq B\right\}$ for $z^{\prime} \in E^{\prime}$. 
Proof. We prove a), the proof of bl is similar.

For $f \in C(K, E)$ and $f(A) \subseteq B$, there is a net $\left(f_{j}\right)$ of E-simple functions which converges uniformly to $f$ and $f_{j}(A) \subseteq B$ for every $j$. Then

$$
q\left(\int_{A} f d m\right)=q\left(\lim \int_{A} f_{j} d m\right) \leq m_{B q}(A) .
$$

On the other hand, for $\varepsilon>0$ there is a partition $\pi \in \Pi(A)$ $\pi=\left\{A_{1}, \ldots, A_{n}\right\}$, a finite set $\left\{x_{1}, \ldots x_{n}\right\} \subseteq B$ and a $z^{\prime} \in V_{q}^{\circ}$ such. that

$$
\tilde{m}_{B q}(A)-\varepsilon<\left.\right|_{i=1} ^{n} \otimes m\left(A_{i}\left|x_{i}, z^{\prime}>\right|=\left|\sum_{i=1}^{n} m_{x_{i} z^{\prime}}\left(A_{i}\right)\right|\right.
$$

For the regularity of $m_{x_{i} z^{\prime}}$, we can choose some compact sets $K_{i} \subset A_{i}$ and disjoint open sets $G_{i} \supseteq K_{i}$, with

$$
\left|m_{x_{i} z^{\prime}}\right|\left(A_{i} \backslash K_{i}\right)<\frac{\varepsilon}{2 n},\left|m_{x_{i} z^{\prime}}\right|\left(G_{i} \backslash K_{i}\right)<\frac{\varepsilon}{2 n} .
$$

(Here $1 . \mid$ denotes the variation of the scalar measure).

Now there are functions $\Psi_{i} \in C(K)$, with $0 \leq \Psi_{i} \leq 1, \Psi_{i}\left(K_{i}\right)=\{1\}$ and $\Psi_{i}\left(K \backslash G_{i}\right)=\{0\}$. Let $f \in C(K, E)$ be

$$
f={\stackrel{n}{\Sigma_{1}}}_{1} x_{i} \Psi_{i}
$$

then

$$
\begin{aligned}
m_{B q}(A) & -\varepsilon<l_{i=1}^{n} m_{x_{i} z^{\prime}}\left(A_{i}\right)-{ }_{i=1}^{n} m_{x_{i} z^{\prime}}\left(K_{i}\right) \mid+l_{i=1}^{n} m_{x_{i} z^{\prime}}\left(K_{i}\right) \\
& -{ }_{i=1}^{n} \int_{A} \Psi_{i} m_{x_{i} z^{\prime}}|+|<\int_{A} f d m, z^{\prime}>\mid<\varepsilon+q\left(\int_{A} f d m\right) .
\end{aligned}
$$

Since $\varepsilon$ is arbitrary, this completes the proof.

Remark: Looking at the above proof, we can deduce, when $A$ is an open set, that: 


$$
\begin{gathered}
\tilde{m}_{B q}(A)=\sup \left\{q\left(\int_{A} f d m\right): f \in C(K, E), f(A) \subset B, \operatorname{supp}(f) \subseteq A\right\} ; \\
\left|m_{z^{\prime}}\right|_{p_{B}}(A)=\sup \left\{\left|<\int_{A} f d m_{,} z^{\prime}>\right|: f \in C(K, E), f(A) \subseteq B, \operatorname{supp}(f) \subset A\right\} .
\end{gathered}
$$

In the next theorem, the equivalence $a \Leftrightarrow d$ gives an interesting characterisation of the operators with S.C.v. representing measure.

THEOREM 5. Let $T: C(K, E) \rightarrow F$ be con operator with representing measure $m: \Sigma \rightarrow L\left(E, F^{\prime \prime}\right)$. Then, the following assertions are equivalent:

a) $m$ is s.c.v.;

b) $\quad m(\Sigma) \subseteq L(E, F)$ and for every bounded disc $B \subseteq E$ and every $q \in P_{F^{\prime}}$ the set of scalar measures $\left\{\left|m_{z},\right|_{p_{B}}: z^{\prime} \in V_{q}^{\circ}\right\}$ is uniformly countably additive;

c) For each $B$ and $q$ as in b) there is a finite positive regular Borel control measure $\mu$ on $K$ such that

$$
\lim _{\mu(A) \rightarrow 0} \tilde{m}_{B q}(A)=0
$$

d) $\left(T f_{n}\right)$ tends to zero for every uniformly bounded sequence $\left(f_{n}\right) \subseteq C(K, E)$ converging pointwise to zero.

Proof: The equivalence $a \Leftrightarrow b \Leftrightarrow c$ follows from (2) and from some classical results for sets of scalar measures (see I. 2 of [4] or IV. 9 of [6]).

$c \Rightarrow d)$ Let $\left(f_{n}\right) \subseteq C(K, E)$ be a uniformly bounded sequence, converging pointwise to zero, we shall prove that $\left(T f_{n}\right)$ tends to zero. Let $B \subseteq E$ be a bounded disc with $f_{n}(K) \subset B$ for every $n$. If $q \in P_{F}$, there is a finite positive regular Borel measure $\mu$ and a $\partial>0$ such that

$$
\tilde{m}_{B q}(A)<\frac{2}{2} \text { when } \mu(A)<\partial
$$

Now let $p \in P_{E}$ satisfy $p \sim q$, then the sequence $\left(p \circ f_{n}\right) \subseteq C(K)$ converges pointwise to zero, so that, by the Egoroff theorem, there is a 
$K_{0} \in \Sigma$, with $\mu\left(K \backslash K_{0}\right)<\partial$, and $n_{0}$ such that

$$
p\left(f_{n}(s)\right)<\frac{1}{2 \tilde{m}(p, q)^{(K)}}
$$

for $s \in K$ and $n>n_{0}$. Then

$$
q\left(T f_{n}\right) \leq q\left(\int_{K_{0}} f_{n} d m\right)+q\left(\int_{K \backslash K_{0}} f_{n} d m\right) \leq \frac{\tilde{m}_{(p, q)}\left(K_{0}\right)}{2 \tilde{m}}(p, q)^{(K)}+\tilde{m}_{B q}\left(K \backslash K_{0}\right)
$$

Hence $q\left(T f_{n}\right)<1$ for $n>n_{0}$ and we conclude that $\left(T f_{n}\right) \rightarrow 0$.

$d \Rightarrow b)$ Since $C(K)$ has the reciprocal Dunford-Pettis property (see [7]), for each $x \in X$ the operator ${ }^{T} x$ is weakly compact, so

$m(\Sigma) \subseteq L(E, F)$ and it suffices to show that for any bounded disc

$B \subset E$ and any $q \in P_{F}$ the family of scalar measures

$\left\{\left|m_{z^{\prime}}\right|_{p_{B}}: z^{\prime} \in V_{q}^{\circ}\right\}$ is uniformly countably additive. Indeed if it were

not, then there is a sequence $\left(z_{n}^{\prime}\right) \subset V_{q}^{0}$, and another $\left(G_{n}\right)$ of

disjoint open sets in $K$, with

$$
\left|m_{z_{n}^{\prime}}\right|_{p_{B}}\left(G_{n}\right)>\epsilon
$$

Now by proposition 4 and its remark, we can choose a sequence of functions $\left(f_{n}\right) \subset C(K, E)$ such that for every $n$ we have

$$
f_{n}(K) \subseteq B, f_{n}\left(K \backslash G_{n}\right)=\{0\}, \quad \mid<\int_{K} f_{n} d m, z_{n}^{\prime}>1>\epsilon
$$

This sequence is uniformly bounded and converges pointwise to zero. However $\left(T f_{n}\right)$ does not converge to zero in $F$ because

$$
q\left(T f_{n}^{\prime} \geq 1<\int_{K} f_{n} d m, z_{n}^{\prime}>1>\epsilon\right.
$$

and this contradicts d). 


\section{Unconditionally converging operators}

In the following, we are going to characterise the unconditionally converging operators from $C(K, E)$ to $F$.

Recall that an operator $T$ between $E$ and $F$ is unconditionally converging if $T$ maps weakly unconditionally Cauchy (w.u.c.) series into unconditionally convergent ones, or, what is equivalent, $\left(T x_{n}\right)$ tends to zero in $F$ when $\Sigma x_{n}$ is a w.u.c. series in $E$.

The next result follows from 14.6 of [8].

- LEMMA 6. For every sequence $\left(x_{n}\right)$ in $E$, the following assertions are equivalent:

a) $\Sigma x_{n}$ is w.u.c.;

b) $\Sigma \mid<x_{n}, x^{\prime}>1<\infty$ for each $x^{\prime} \in E^{\prime}$;

c) $\left\{\sum_{n \in M} x_{n}: M \in E(\mathbb{I N})\right\}$ is a bounded set in $E$.

Here $F(\mathbb{I N})$ denotes the system of alz finite subsets of $\mathbb{I N}$.

THEOREM 7. Let $T: C(K, E) \rightarrow F$ be an unconditionally converging operator, then its representing measure $m$ satisfies

a) $m$ is s.c.v.;

b) for every $A \in \Sigma, m(A): E \rightarrow F$ is an unconditionally converging operator.

Proof. The proof of a) is just like that of $" d \Rightarrow b "$ in Theorem 5, since the sequence $\left(f_{n}\right)$ mentioned there satisfies:

i) $\left\{\sum_{n \in M} f_{n}: M \in F(\mathbb{N})\right\}$ is a bounded set in $C(K, E)$. So $\Sigma f_{n}$ is a w.u.c. series;

ii) $\left(T f_{n}^{\prime}\right.$ does not converge to zero.

b) Suppose that $T$ is an unconditionally converging operator, $A \in \Sigma$ and $\sum x_{n}$ a w.u.c. series in $E$. We shall prove that $\left(m(A) x_{n}\right)$ tends to zero in $F$. 
Let $B$ be a bounded disc in $E$ such that $\left\{x_{n}: n \in \mathbb{N}\right\} \subseteq B$. If $q \in P_{F}$, using the existence of a regular control measure for $\tilde{m}_{B q}$, we can find a compact $H$ and an open $G$ in $K$ with $H \subseteq A \subseteq G$ and $\tilde{m}_{B q}(G \backslash K)<\frac{1}{2}$, then there is a function $\Psi \in C(K)$ such that $0 \leq \Psi \leq 1$, $\Psi(G \backslash H)=\{0\}$ and $\Psi(H)=\{1\}$. We define $f_{n} \in C(K, E)$ by $f_{n}=x_{n}^{\Psi}$, it is clear that $\Sigma_{f_{n}}$ is a w.u.c. series, so $\left(T f_{n}\right)$ tends to zero and we have

$$
q\left(T f_{n}-m(A) x_{n}\right)=q\left(\int_{K}\left(\Psi-x_{A} x_{n}\right) d m\right) \leq \tilde{m}_{B q}(G \backslash H)<\frac{1}{2} .
$$

Therefore we obtain that $q\left(m(A) x_{n}{ }^{\prime}<1\right.$ for almost every $n$. Hence $\left(m(A) x_{n}\right)$ converges to zero and the proof is complete.

An immediate consequence of Teorems 5 and 7 is:

COROLLARY 8. If $T: C(K, E) \rightarrow F$ is an unconditionally converging operator, then $\left(T f_{n}\right)$ tends to zero for every uniformly bounded sequence $\left(f_{n}\right)$; $C(K, E)$ converging pointwise to zero.

The converse of the above result is not true in general. Now, we characterise those spaces $E$ for which this converse holds.

DEFINITION 9. A locally convex space $E$ is weakly $\Sigma$-complete if every w.u.c. series in $E$ is weakly convergent.

All the weakly sequentially complete spaces, and so all the semireflexive ones, are weakly $\Sigma$-complete. An easy extension of the BessagaPelczynski theorem proves that a sequentially complete locally convex space $E$ is weakly $\Sigma$-complete if and only if it does not contain a copy of $C_{0}$.

If $E$ is a weakly $\Sigma$-complete space, the converse of corollary 8 is true; furthermore this property characterises the weakly $\Sigma$-complete spaces, as we prove in the next theorem.

THEOREM 10. The following assertions are equivalent;

a) $E$ is weakly $\Sigma$-complete; 
b) for any compact Hausdorff space $K$ and any space $F$, an operator $T: C(K, E) \rightarrow F$ is unconditionally converging if and only if its representing measure is s.c.v.;

c) there is a compact $K$ such that every operator $T$ from $C(K, E)$ to $E$ with representing measure s.c.v. is unconditionally converging.

Proof. a $\Rightarrow$ b) Let $\Sigma f_{n}$ be a w.u.c. series, then $\sum f_{n}(t)$ is weakly convergent for every $t \in K$, then, according to the orlicz-Pettis theorem, $\Sigma f_{n}(t)$ is convergent for each $t$. Therefore $\left(T f_{n}\right)$ tends to zero in $F$, because $\left(f_{n}\right)$ is a uniformly bounded sequence converging pointwise to zero in $C(K, E)$ and $m$ is s.c.v.

$b \Rightarrow c)$ Trivial.

$\mathrm{c} \Rightarrow \mathrm{a})$ First we fix $a \in K$ and define an operator $T$ on $C(K, E)$ by $T(f)=f(a)$. Then, by Theorem 5 , the representing measure of $T$ is s.c.v., so $T$ is unconditionally converging.

Now we consider a function $\Psi \in C(K)$ with $0 \leq \Psi \leq 1$ and $\Psi(\alpha)=1$. If $\Sigma x_{n}$ is a w.u.c. series in $E$, then $\sum f_{n}$, with $f_{n}=x_{n}{ }^{\Psi}$ is w.u.c. in $C(K, E)$, so $\Sigma T\left(f_{n}\right)=\Sigma x_{n}$ is unconditionally convergent in $E$. Hence $E$ is weakly $\Sigma$-complete.

The result $" b \Rightarrow a$ " of the above theorem extends, with an easier proof, an analogous theorem proved by Saab in [9] for $E$ and $F$ Banach spaces.

Bombal and Cembranos show in [2] that conditions a) and b) in theorem 7 characterise the unconditionally converging operators from $C(K, E)$ to $E$, for $E$ and $F$ Banach spaces, if and only if $K$ is a dispersed compact (that is, it does not contain any perfect set). In our case this result is also true.

THEOREM 11. Let $K$ be a dispersed compact and $T$ an operator from $C(K, E)$ to $F$, with representing measure $m$, then the following assertions are equivalent: 
a) $T$ is an unconditionally converging operator

b) $m$ is s.c.v. and for each $A \in \Sigma, m(A): E \rightarrow F$ is an unconditionally converging operator.

Proof. The proof of $" b \Rightarrow a n$ is similar to that of Theorem 7 of [2], but we use that for a regular Borei measure $\mu$ in a dispersed compact $K$ there is a countable family $\left(x_{n}\right)$ in $K$ such that

$$
\mu=\sum_{n \in \mathbb{N}} \mu\left(x_{n}\right) \partial_{x_{n}}
$$

(see [10] p.338) instead, to consider a metrisable quotient of $K$.

Remark: It is also possible to prove an analogue of the previous theorem for compact and weakly compact operators from $C(K, E)$ to $F$.

\section{References}

[1] J. Batt and E.J. Berg, "Linear bounded transformations on the space of continuous functions", J. Funct. Anal. 4 (1969), 215-239.

[2] F. Bombal and P. Cembranos, "Characterization of some classes of operators on spaces of vector-valued continuous functions", Math. Proc. Cambridge Philos. Soc. 97 (1985), 137-146.

[3] J.K. Brooks and P.W. Lewis, "Linear operators and vector measures", Trans. Amer. Math. Soc. 192 (1974), 139-163.

[4] J. Diestel and J.J. UhI, Vector measures, (Math. Surveys 15 A.M.S. Providence R.I. 1977).

[5] I. Dobrakov, "On representation of linear operators on $C_{0}(T, X)$ ", Crechoslovak Math. J. 21 (1971), 13-30.

[6] N. Dunford and J.T. Schwartz, Linear operators, part I (Interscience, New York, London 1958).

[7] A. Grothendieck, "Sur les applications linéaires faiblement compactes d'espaces du tipe $\mathrm{C}(\mathrm{K})$, Conad. J. Math. 5 (1953), 129-173.

[8] H. Jarchow, Locally convex spaces, (Teubner, Stuttgart 1981).

[9] P. Saab, "Weakly compact, unconditionally converging and DunfordPettis operators on spaces of vector valued continuous functions", Math. Proc. Cọmbridge Philos. Soc. 95 (1984), 101-108. 
[10] 2. Semadeni, Banach spaces of continuous functions, (PWN-Polish Scientific Publishers, Warszawa 1971).

[11] A.H. Shuchat, "Integral representation theorems in topological vector spaces", Trons. Amer. Math. Soc. 172 (1972), 373-397.

[12] K. Swong, "A representation theory of continuous linear maps", Math. Ann. 155 (1964) 270-291.

Departamento de Teoria de Funciones,

Facultad de Ciencias Matematicas,

Universidad Complutense de Madrid,

28040 Madrid,

Spain. 